\title{
DOSIS LETALES DE DIPTEREX 80 \% P.S PARA EL CONTROL DE NAYADAS DE Gomphaeshna sp., Tramea cophysa y Tramea calverti (Odonata, Aeshnidae)
}

Fernando Alcántara B. ${ }^{1}$, Juan García T. ${ }^{1}$, Palmira Padilla P. ${ }^{1}$ y César Delgado V. ${ }^{1}$

\section{RESUMEN}

Las nayadas de Gomphaeshna sp., Tramea cophysa y Tramea calverti (Odonata), fueron manipuladas en laboratorio para establecer la dosis letal $100 \%$ en períodos de 24 horas de tratamiento, con el insecticida fosforado Dipterex 80 \% Polvo Soluble ("Trichlorfon"), 0,0 dimetil (2,2,2-triclor-1-hidroximetil) fosfonato.

Se efectuó un experimento para Gomphaeshna sp. y otro para Tramea cophysa y Tramea calverti, en acuarios de vidrio, con concentraciones crecientes del insecticida.

Para Gomphaeshna sp., se estableció una DL100 en 24 horas de 0,5 mg/l y para Tramea cophysa y Tramea calverti se estableció una DL100 de 4,5 mg/l.

Palabras clave: Dosis letales de Dipterex. Nayadas de Gomphaeshna sp., Tramea cophysa y Tramea calverti. Odonata.

\begin{abstract}
The naiads of Gomphaeshna sp., Tramea cophysa and Tramea calverti (Odonata), were manipulated in a laboratory to determine 24 LD100 of the phosphonate - based insecticide Dipterex 80 \% P.S. (Trichlorfon) 0,0 dimetyhyl (2,2,2 - trichlor - 1 methylhydrox) phosphonate.

One experiment were done for Gomphaeshna sp. and one for T. cophysa and $T$. calverti in glass aquaria with increasing amounts of the insecticide.

1 Instituto de Investigaciones de la Amazonía Peruana - IIAP. Programa de Investigación de Ecosistemas Acuáticos. Centro Regional de Investigaciones de Loreto. Av. Abelardo Quiñones km. 2,5. Apartado 784 Fax 265527. Teléfonos 265515/265516.
\end{abstract}


The 24 DL100 for Gomphaeshna sp. was established at $0,5 \mathrm{mg} / 1$ and at 4,5 $\mathrm{mg} / 1$ for T. cophysa and T. calverti.

\section{INTRODUCCIÓN}

Las nayadas de Odonatos son abundantes en los ambientes de cultivo de peces en el área de Iquitos, constituyendo un problema en los estanques de larvicultura, por la alta predación de larvas y alevinos de gamitana (Colossoma macropomum), paco (Piaractus brachypomus), y boquichico (Prochilodus nigricans) producidos en condiciones controladas. (Alcántara, 1985; Guerra y Alcántara, 1992; Alcántara 1993).

Sinha y Roy (1991), estudiando la composición de especies y las variaciones estacionales de los insectos acuáticos en dos estanques, con y sin manejo, en la India, determinaron que las larvas/ninfas y adultos de Coleóptera y Hemíptera son predadores de larvas y alevinos de peces, en tanto que, en el caso de Odonata lo son sólo las ninfas. Estos autores determinaron, asimismo, que la abundancia de insectos predadores fue mayor en el estanque sin manejo.

Delgado et al. (1994), reportaron que la densidad de nayadas de Odonatos en un estanque de piscicultura, sin manejo, en el área de Iquitos, Perú, tiene una correlación positiva altamente significativa, con el tiempo de llenado del estanque $(r>0,97)$ y con la presencia de macrófitas, llegando a declinar notoriamente cuando se extrae la vegetación.

Este trabajo se efectuó con la finalidad de determinar la dosis letal mínima del insecticida Dipterex 80 \% P.S., para controlar las nayadas de las especies de Odonatos dominantes Gomphaeshna sp., Tramea cophysa y T. calverti, en los estanques de larvicultura.

\section{MATERIAL Y MÉTODOS}

Los experimentos se realizaron en el Centro Regional de Investigación de Loreto del IIAP, en Quistococha, en el período comprendido entre junio y agosto de 1996.

Se utilizaron acuarios de vidrio de 70 x 40 x $40 \mathrm{~cm}$. En cada acuario se colocó cinco litros de agua y cinco nayadas de las especies problema, distribuidas al azar. 
Antes de la aplicación del insecticida se sometió a aclimatación a las nayadas, durante un período de 24 horas, proporcionándose como alimento insectos acuáticos, Corixidae (Díptera).

Se estableció un experimento para Gomphaeshna sp. y otro para Tramea cophysa y Tramea calverti, tratadas conjuntamente. Cada experimento se efectuó con tres repeticiones.

La determinación de la dosis letal 100\% (DL100) se efectuó en períodos de 24 horas.

\section{RESULTADOS Y DISCUSIÓN}

Tabla 1. Dosis letal para Gomphaeshna sp . en 24 horas.

\begin{tabular}{|c|c|c|c|c|}
\hline \multirow{2}{*}{$\begin{array}{c}\text { Número de } \\
\text { acuario }\end{array}$} & \multirow{2}{*}{ Dosis (mg/l) } & \multirow{2}{*}{$\begin{array}{c}\text { Número de } \\
\text { nayadas }\end{array}$} & \multicolumn{2}{|c|}{ Mortalidad } \\
\cline { 3 - 5 } & & & Número & \% \\
\hline 1 & 0,2 & 5 & 1 & 20 \\
\hline 2 & 0,2 & 5 & 3 & 60 \\
\hline 3 & 0,2 & 5 & 0 & 0 \\
\hline 4 & 0,3 & 5 & 3 & 60 \\
\hline 5 & 0,3 & 5 & 1 & 20 \\
\hline 6 & 0,3 & 5 & 5 & 100 \\
\hline 7 & 0,4 & 5 & 5 & 100 \\
\hline 8 & 0,4 & 5 & 4 & 80 \\
\hline 9 & 0,4 & 5 & 2 & 40 \\
\hline 10 & 0,5 & 5 & 5 & 100 \\
\hline 11 & 0,5 & 5 & 5 & 100 \\
\hline 12 & 0,5 & 5 & 5 & 100 \\
\hline 13 & 0,0 & 5 & 0 & 0 \\
\hline 14 & 0,0 & 5 & 0 & 0 \\
\hline 15 & 0,0 & 5 & 0 & 0 \\
\hline
\end{tabular}


Figura 1. Dosis letal de Dipterex 80 \%.S. en 24 horas para Gomphaeshna sp.

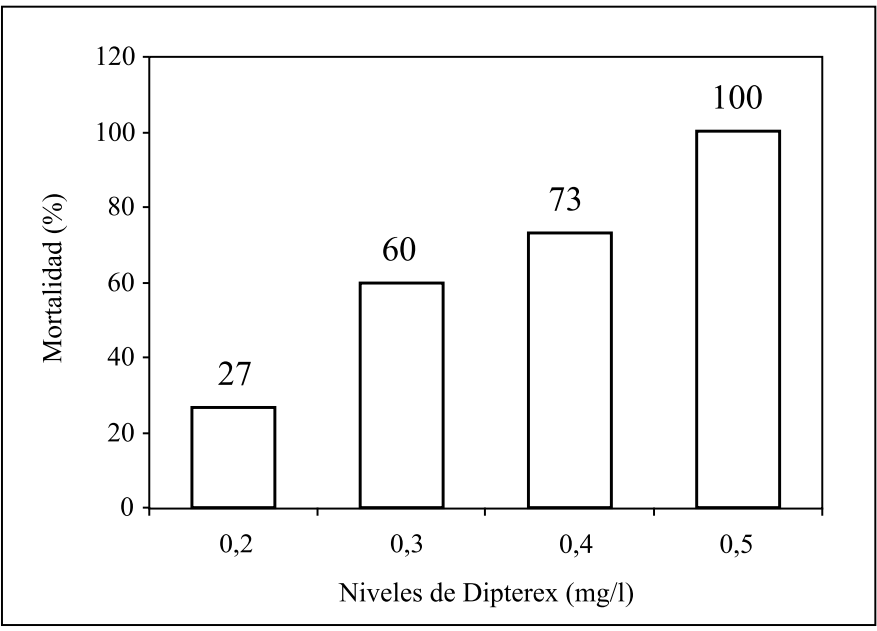

Tabla 2. Dosis letal para Tramea cophysa y Tramea calverti en 24 horas.

\begin{tabular}{|c|c|c|c|c|}
\hline \multirow{2}{*}{$\begin{array}{c}\text { Número de } \\
\text { acuario }\end{array}$} & \multirow{2}{*}{ Dosis (mg/l) } & \multirow{2}{*}{$\begin{array}{c}\text { Número de } \\
\text { nayadas }\end{array}$} & \multicolumn{2}{|c|}{ Mortalidad } \\
\cline { 4 - 5 } & & & Número & $\%$ \\
\hline 1 & 0,5 & 5 & 0 & 0 \\
\hline 2 & 0,5 & 5 & 0 & 0 \\
\hline 3 & 0,5 & 5 & 0 & 0 \\
\hline 4 & 1,0 & 5 & 0 & 0 \\
\hline 5 & 1,0 & 5 & 0 & 0 \\
\hline 6 & 1,0 & 5 & 0 & 0 \\
\hline 7 & 1,5 & 5 & 0 & 0 \\
\hline 8 & 1,5 & 5 & 0 & 0 \\
\hline 9 & 1,5 & 5 & 0 & 0 \\
\hline 10 & 2,0 & 5 & 0 & 0 \\
\hline 11 & 2,0 & 5 & 0 & 0 \\
\hline 12 & 2,0 & 5 & 0 & 0 \\
\hline 13 & 2,5 & 5 & 0 & 0 \\
\hline 14 & 2,5 & 5 & 0 & 0 \\
\hline 15 & 2,5 & 5 & 0 & 0 \\
\hline 16 & 3,0 & 5 & & 0 \\
\hline
\end{tabular}


DOSIS LETALES DE DIPTEREX 80\% P.S. PARA EL CONTROL DE NAYADAS DE

\begin{tabular}{|r|r|r|r|r|}
\hline 17 & 3,0 & 5 & 0 & 0 \\
\hline 18 & 3,0 & 5 & 2 & 40 \\
\hline 19 & 3,5 & 5 & 5 & 100 \\
\hline 20 & 3,5 & 5 & 5 & 100 \\
\hline 21 & 3,5 & 5 & 4 & 80 \\
\hline 22 & 4,0 & 5 & 4 & 80 \\
\hline 23 & 4,0 & 5 & 5 & 100 \\
\hline 24 & 4,0 & 5 & 3 & 60 \\
\hline 25 & 4,5 & 5 & 5 & 100 \\
\hline 26 & 4,5 & 5 & 5 & 100 \\
\hline 27 & 4,5 & 5 & 5 & 100 \\
\hline 28 & 0,0 & 5 & 0 & 0 \\
\hline 29 & 0,0 & 5 & 0 & 0 \\
\hline 30 & 0,0 & 5 & 0 & 0 \\
\hline
\end{tabular}

Figura 2. Dosis letal de Dipterex 80 \% P.S. en 24 horas para Tramea cophysa y T. calverti .

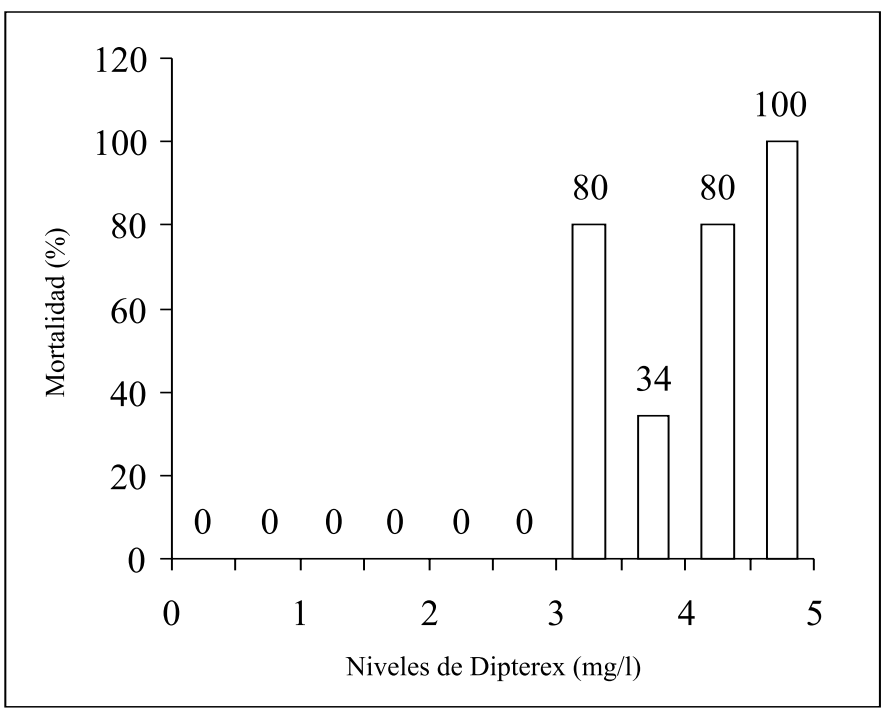


Las nayadas de Gomphaeshna sp. se caracterizan por su gran tamaño, que llega hasta los cinco centímetros y por su rápido desplazamiento en los ambientes acuáticos, debido a que incorporan grandes cantidades de agua en el abdomen y la expulsan en forma de chorros intermitentes hacia atrás. Esta forma de propulsión a chorro les da una buena capacidad de desplazamiento y, a la vez, las hace más vulnerables al insecticida aplicado, alcanzándose la dosis letal con niveles relativamente bajos, de 0,5 mg/l. Fig. 1 .

Las nayadas de Tramea cophysa y T. calverti presentan porte pequeño, que no sobrepasa los dos centímetros, y no incorporan agua en el abdomen como lo hace Gomphaeshna sp. Por esta razón, presentan menor sensibilidad al insecticida, requiriendo por tanto niveles más altos, de hasta $4,5 \mathrm{mg} / \mathrm{l}$ para alcanzar la dosis letal $100 \%$ en 24 horas. Fig. 2.

Ambas especies conviven en los estanques, llegando a alcanzar densidades considerables, que Delgado et al (1995) estimaron en $415 / \mathrm{m}^{2}$, en el área de Iquitos; por lo cual tienen alta incidencia en la predación de larvas de peces, debido a su régimen carnívoro, Roy (1990) y Sinha y Roy (1991).

Las dosis se han establecido en acuarios de vidrio, por tanto falta validar estos resultados en estanques, determinando, a la vez, los efectos residuales del insecticida y su inocuidad sobre las larvas o alevinos de peces. 


\section{BIBLIOGRÁFICAS}

ALCÁNTARA, F. 1985. Reproducción inducida de "gamitana," Colossoma macropomum (Cuvier, 1818), en el Perú. Tesis Doctoral. Universidad Nacional de Trujillo. Mimeografiado. 38 pp.

ALCÁNTARA, F. 1993. Estado del cultivo de gamitana, Colossoma macropomum, y paco, Piaractus brachypomus, en el Perú. Red Latinoamericana de Acuicultura. CIID/COLCIENCIAS. 35 pp. No publicado.

DELGADO, C.; F. ALCÁNTARA Y G. COUTURIER. 1994. Densidad de larvas de Odonatos (Insecta) en un estanque de piscicultura en Iquitos. Rev. per. Ent. 37:101-102.

GUERRA, H. ; F. ALCÁNTARA; H. SÁNCHEZ Y S. ÁVALOS. 1,992. Hibridación de "paco", Piaractus brachypomus, (Cuvier, 1818) x "gamitana", Colossoma macropomum, (Cuvier, 1818), en Iquitos, Perú. Folia Amazónica 4(1):103-110.

ROY, SP. 1990. Evaluation on the exploitation of fish-pond organisms by certain predatory insects in an aquaculture pond at Bhagalpur. J. FRESHWAT. BIOL.; vol. 2, no. 3, pp. 257-264.

SINHADK; ROY SP. 1991. Species composition and seasonal abundance of predatory insects in some fish culture ponds at Dumka (India). J. FRESHWAT. BIOL.; vol. 3, no. 1, pp. 99-103. 